\title{
M-Advertising: otra forma de pensar el marketing y su impacto En la relación uso/consumo
}

\author{
Hedilberto Granados LóPEZ ${ }^{1}$ \\ Universidad Católica de Manizales
}

Recibido: 29/04/2014

Aceptado: 26/05/2014

"Es un hecho aceptado por todos que las nuevas tecnologías están transformando tanto los hábitos y necesidades del consumidor como los planes de negocio de las campañas"

Mónica Deza

\section{Resumen}

Las acciones comerciales muestran en la actualidad que las plataformas móviles o nuevas pantallas representan una manera efectiva y estratégica para incentivar en las personas la necesidad de comunicar e informar de manera inmediata sus necesidades de consumo. Así pues, el acto comunicativo desde la perspectiva de la comunicación publicitaria se establece desde un enfoque destinado a la persuasión y consumo estratégico de bienes y servicios. De ahí, que la comunicación publicitaria tenga en parte por objeto, la optimización de las posibles formas para pensar el marketing dentro de un núcleo de acción personal a través de la comunicación intencional de marcas de modo simbólico, o por lo que se podría advertir en el caso de mobile marketing, la puesta en escena de una acción de tipo comercial canalizada a través de un móvil o una pantalla, cuyo objetivo central es la persuasión directa para el consumo de bienes y servicios dentro del marco de un movimiento del marketing que hace evidente, la transformación de los vehículos de acción del mundo del marketing.

Palabras clave: uso/consumo, Marketing, Mobile marketing, nuevas pantallas.

${ }^{1}$ Profesor Investigador de la Facultad de Ciencias Sociales y Administración de la Universidad Católica de Manizales (Colombia) E-mail: egranados@ucm.edu.co. El presente artículo hace parte de una investigación sobre "uso y consumo de comunicación publicitaria en jóvenes universitarios de la ciudad de Manizales (Colombia) llevada a cabo por el grupo de investigación en marca publicitaria Altamira y patrocinado por el Universidad Católica de Manizales (Colombia) 


\title{
M-Advertising: another way of thinking about marketing and its impact In the usage/consumption ratio
}

\begin{abstract}
Commercial actions today show that mobile platforms or new screens represent an effective and strategic way to encourage people the need to communicate and inform immediately your consumption needs. The Act of communication from the perspective of advertising communication is thus establishes an approach aimed at persuasion and strategic consumption of goods and services. Hence, that advertising aims in part, establish the possibility of optimization of possible ways to think about the marketing within a core of personal action through intentional communication of symbolic mode marks, or what could notice in the case of mobile marketing, the staging of a commercial type channeled through a mobile phone or a screen action whose central objective is to direct persuasion for the consumption of goods and services within the framework of a movement of marketing that makes evident, the transformation of the action in the world of marketing vehicles.
\end{abstract}

Keywords: use/consumption, Mobile marketing, new screens.

\section{Introducción}

Uno de los retos que en la actualidad enfrenta el marketing para hacer frente a las transformaciones del marketing tradicional en M-Advertising o Mobile marketing ${ }^{2}$ radica, en comprender de manera estratégica el lapso o transición en el cual, la forma clásica de producción publicitaria pensada desde los medios de grandes audiencias o mass media (MCM), inició un tránsito hacia un fenómeno individual denominado social media ${ }^{3}$.

Este tránsito hacia los medios o redes sociales ofrece no sólo un nuevo enfoque comunicativo, sino otras formas de pensar lo estratégico presente en el marketing, que siempre ha tenido por lugar común el mensaje publicitario como ese juego intencionado entre la realidad y la ensoñación como lo manifiesta San Nicolás Romera ${ }^{4}$ al hablar de las campañas publicitarias; pero esta vez, pensado a partir de su personalización y a través, de la utilización de dispositivos móviles bombardeados con múltiples pautas, que intentan llegar a todos las redes sociales como Facebook, twiter, badoo o motores de búsqueda como google, yahoo o alta vista etc, lo que hace que los potenciales clientes, estén continuamente en contacto directo con una gran variedad de mensajes destinados no tanto al ofrecimiento de un producto en sí, sino a la utilidad y al servicio que de éste se pueda desprender en contraste, con su valor de cambio el cual, se constituye como punto de encuentro entre lo que se desea transmitir como un servicio y el ofrecimiento comercial hacia el sujeto/objeto del mensaje. En consecuencia, y al decir de Del Pino 5 "este nuevo escenario permite vislumbrar un alud de experiencias de comunicación,

2 La cursiva es mía.

${ }^{3}$ El término "Social Media" es usado como contraste del conocido "Mass Media" para expresar el enorme cambio de paradigma que están viviendo los medios de comunicación en la actualidad. La mayoría de las veces el término es usado para referirse a actividades que integran la tecnología, las telecomunicaciones móviles y la interacción social, en forma de podcast, vodcast, MMS, SMS y WAP entre muchos otros.

${ }^{4}$ Romera San N. C. (2004): "Publicidad Corporativa y Cultura Cotidiana" http://www. comminit.com/la/node/150542 Web visitada el 28/05/2014.

${ }^{5}$ Del Pino, R, C. (2010): "La Internet y los Nuevos Consumidores: el Nuevo Modelo Publicitario" Revista telos Dossier, 57, 55-82. 
donde lo más relevante es la creación de un mensaje tan preciso y atractivo que deja de ser una molestia"

En este sentido Gomez $z^{6}$ plantea que:

Los anunciantes comienzan a ver con gran interés los medios de comunicación móviles como las nuevas plataformas de difusión publicitaria: teléfonos móviles, PDAs, iPods (...) Estos nuevos soportes hacen necesaria una redefinición y análisis del marketing para estas tecnologías de nueva generación

Esta movilidad del objeto como bien de adquisición al servicio que se deriva del mismo, está marcando un nuevo enfoque para pensar la comunicación publicitaria a través de vías alternativas más eficientes y económicas de pauta como los móviles o las denominadas nuevas pantallas. Así pues, la pauta en redes sociales destinada al comercio de bienes y servicios empieza a tomar fuerza dentro de las estrategias de marketing en el entorno mundial de los mercados digitales de ahí, que en los denominados objetivos de marketing y objetivos publicitarios, se tienda cada vez más a la creación, unión y despliegue de campañas cuyo objetivo fundamental está en generar lanzamientos de negocios a largo plazo como lo plantea Gonzales Oñate ${ }^{7}$ en el caso del lanzamiento de una marca.

En consecuencia, y para adentrarse un poco más en la comprensión de este reciente fenómeno denominado por sus plataformas de acción M-Adversiting, se pasará a realizar una exposición general de sus principales características y alcances.

\section{El mobile marketing y los mercados interactivos}

El M-Advertising o mobile marketing ${ }^{8}$, en su acepción más básica es el uso de dispositivos de comunicación portátil tales como celulares smartphone, ipad, ipod, tables entre otras formas tecnológicas de comunicación e interacción personales para la transmisión de mensajes con fines comerciales y para los cuales, se ha generando toda una plataforma de mensajes y formas digitales de agenciamiento publicitario de rápido cambio y acceso, en los que el anuncio como lo manifiesta Gomez Rosado9 "ha dejado de ser una quimera rectilínea para convertirse en un debate curvilíneo con su audiencia". Este, giro de lo rectilíneo a lo curvilíneo supuesto en las nuevas tecnologías centradas en el aspecto publicitario y comunicativo radica, de cierto modo, en haber superado las barreras físicas de comunicación y oferta a través de la virtualización del mercado.

\footnotetext{
${ }^{6}$ Gomez, T, A. (2010): “El Mobile Marketing como Estrategia de Comunicación” Revista Icono14, 240, 238-260.

7 GonZales OÑate, C. (2008) "Estrategia de Comunicación Publicitaria para el Lanzamiento de una Marca" Universitat de Jaune, 4, 2-33.

${ }^{8}$ El Mobile Marketing hace referencia al tipo de publicidad generada de y para dispositivos telefónicos móviles como celulares, ipod, ipad, tables etc.

9 Gomez Rosado, D, (2004) "Publicidad Interactiva" Revista Mosaic, tecnologías y comunicación multimedia, 6, 3-31.
} 
De acuerdo $A \operatorname{costa}^{10}$, esto ha llevado a que en lo últimos años, se haya generado entre los publicistas y anunciantes un interés creciente sobre las posibilidades de los nuevos medios y soportes digitales como sistemas de difusión publicitaria. Por lo que ésta nueva dinámica, ha terminado impactando, el modo y la percepción comunicativa de los usuarios y por ende, la manera cómo éstos perciben y se relacionan con el mercado ya no a través de productos, sino de los servicios que éstos prometen.

Este paso, de productos a servicios manifiesto en el mobile marketing y pese a que sea un fenómeno relativamente nuevo dentro del imaginario publicitario, hace que surja la necesidad de ofrecer una mirada estructural de esta nueva estrategia de publicitación digital que permita comprender en qué sentido o medida se están alterando las percepciones de los consumidores. Esta primera comprensión resultará útil en la medida que posibilita valorar las dimensiones del cambio tanto cualitativo, como cuantitativo entre las relaciones del mercado inmerso en los nuevos modelos digitales, y las nuevas formas de agenciamiento de la comunicación publicitaria en su mediación básica. Es decir, en la venta de servicios.

En este sentido y de cuerdo a la necesidad planteada de esclarecer una primera estructura de este cambio en la comunicación publicitaria, se partirá de la forma en qué se están estructurando y transformando el mercado de los mensajes con fines publicitarios. Así pues, y para entrar en materia, al hablar de Mobile Marketing, se hace alusión específicamente al envío de múltiples formatos de mensajes publicitarios cuyo medio de transmisión directa son los dispositivos de comunicación móvil, dentro de los cuales, se debe estimar la naturaleza tecnológica de los mismos así, como el rango de uso instrumental o expresivo que le pueda ser atribuido.

Esto traducido en la relación Mobile Marketing da como resultado, que el valor que le sea asignado de fabrica fluctuará de acuerdo a la intención y posibilidad misma para la recepción de mensajes y la posibilidad misma de generar un impacto de consumo en el comportamiento del sujeto/audiencia. Valor, que determinará estratégicamente, tanto el uso instrumental como simbólico del dispositivo celular y de las nuevas pantallas en su relación con los mensajes publicitarios que pueda soportar.

Así pues, el éxito del mensaje publicitario está determinado por las posibilidades de los dispositivos móviles así, como por las aplicaciones que presentan y en dependencia del público al que se desea llegar y las necesidades que se pretendan imponer. Todo ello pensado, dentro de una mirada renovada del uso y estructura del mensaje publicitario en el que ya no se busca la descripción de las bondades de un producto, sino las ventajas simbólicas de su adquisición. Así pues, "en la saturación de los medios y de los mercados en el que se desenvuelven hoy día la mayor parte de las empresas, se busca acudir en auxilio de estrategias publicitarias de tipo emocional en un intento de huir del anonimato de sus anuncios" Ceruelo Ruiz y Gutierrez Arranz ${ }^{11}$, estas nuevas formas de asumir la comunicación publicitaria puede entonces entenderse desde nuevas lógicas de conexión-desconexión con los aparatos, como lo plantea Barbero y

${ }^{10}$ Acosta, Martinez, S. (2006) "La publicidad móvil. El marketing en los nuevos medios". Publicidad y Marketing, IX congreso IBERCOM. Sevilla-Cádiz, 2, 1-9.

11 Ceruelo, C Y Gutiertez, A. (2003): "Eficacia de la publicidad emocional. Un estudio comparativo entre la ejecución de tipo emocional e informativa". Repositorio documental Gredos. 
Téllez ${ }^{12}$ por lo que es factible, encontrarse con nuevos prototipos de interacción social dentro del inventario tecnológico móvil los cuales, se pueden clasificar de acuerdo a cuatro tipologías las cuales, se encuentran asociadas a su vez a un formato determinado de mensaje publicitario con el cual se busca identificar necesidades particulares del consumidor con el fin de diseñar, valorar, comunicar y distribuir los productos que satisfagan mejor tales necesidades. En consecuencia, y de acuerdo a las necesidades previstas en los consumidores, se hace un diseño de los mensajes dentro del contexto publicitario que puedan impactar de manera favorable al consumidor dentro de un rango inicial como se expondrá más adelante.

Dentro de este diseño de mensajería dispuesta a cubrir necesidades particulares del consumidor se puede hacer una división de acuerdo a la intención publicitaria y según el soporte tecnológico para la cual es pensada la campaña publicitaria misma, por lo que este enfoque de acuerdo a Carrillo ${ }^{13}$, sugiere un cambio tangencial en el nuevo estilo de vida interactivo que ha terminado alterando como ya se ha insistido, en la manera como se compra, se interactúa y se da las relaciones con las nuevas tecnologías.

Se debe tener en cuenta que tanto el mensaje publicitario como el soporte para el cual es diseñado el mensaje, parte de una caracterización inicial en la cual, se hace explícito el medio y la mediación de acuerdo a la naturaleza o finalidad de la campaña, así como de las posibles aplicaciones que para la misma se tenga en cuanto al uso instrumental, expresivo o simbólico del medio y el comportamiento del potencial consumidor, y de éstos, frente al impacto del mensaje publicitario y de la plataforma móvil en el que se piense soportar. Así pues, dicha caracterización apela a la creación de tipologías de lo que podría denominarse como nuevos consumidores mediados entre la intención del mercado productor y lo que éste como consumidor aspira obtener. De acuerdo a Galán ${ }^{14}$, en internet y los nuevos consumidores "la denominación empleada para aludir a este tipo de consumidor es la de crossumer o prosumer"

\section{La telefonía celular en el umbral de una nueva lógica comunicacional}

A continuación se presenta una clasificación general de la tipología que abarca los conceptos de nuevos consumidor desde la perspectiva del crossumer y del prosumer antes mencionadas. Para dicha descripción, se tomará como referente un estudio realizado en el año 2003 por the mobile marketing association ${ }^{15}$; estudio en el que se hace una taxonomía del mensaje publicitario vías nuevas tecnologías y pantallas de acuerdo a: característica, naturaleza de campaña, soporte y aplicación discriminadas así:

12 Barbero, M Y Tellez, M. P. (2006): "Los Estudios de Recepción y Consumo en Colombia“. Revista Diálogos de Comunicación. 61, 57-69.

13 Carrillo, M. (2005): "La Interactividad: un Reto para la Publicidad en el Entorno Digital" Revista Zer. 13, 9-24.

${ }^{14}$ Galán, E Y PinO, C. (2010): "Internet y los nuevos consumidores. El nuevo modelo publicitario". Revista de pensamiento sobre comunicación, tecnología y sociedad Telos, 81, 80-96.

${ }^{15}$ El estudio se tituló "A code of conduct and guidelines to best practice" realizado en el año 2003 por The Mobile Marketing Association (MMA). 
- De acuerdo a la tipología de los dispositivos móviles

- De acuerdo a la naturaleza de la campaña publicitaria

- De acuerdo a los soportes del medio comunicativo utilizado para la campaña

- De acuerdo a su aplicación e intención publicitaria

\subsection{De acuerdo a la tipología de los dispositivos móviles}

De acuerdo a la tipología del dispositivo, los teléfonos móviles han sido diseñados bajo la premisa o necesidad de estar en comunicación continua con un determinado grupo de personas, sea éste, familia, círculo social, o laboral. Inicialmente la comunicación se establecía sólo como una relación bidireccional de códigos de voz y texto. Sin embargo, con el desarrollo de mejores plataformas de comunicación y la sofisticación de dispositivos que las pudieran optimizar se ha venido dando una revolución no solo de tipo comunicacional sino también, de orden comercial en la manera cómo se están usando y consumiendo las tecnologías y en lo que a través de ellas se media al punto, como lo plantea Perez Tornero ${ }^{16}$ de hablarse de una interfaz digital con la que los individuos interactúan inmersos en una burbuja digital. De ahí, que la experiencia de la comunicación digital obedezca a cierto giro representacional no sólo sobre la manera en la que se asumen los mercados, sino también de orden cultural en donde, la cultura debe entenderse desde la dimensión del consumo y el intercambio de bienes valores, tal como lo plantea Piñuel ${ }^{17}$ :

Lo que concierne al sistema de objetos de referencia, tanto los actores como los medios de producción y sus productos (...) son los modelos de representación, los cuales pueden constituir "objetos" frente a los cuales se pueden mantener o adoptar actitudes y desarrollar comportamientos reactivo-adaptativos.

Por lo que al referir la comunicación publicitaria a través de los dispositivos móviles, en últimas se está aludiendo no solo a un fenómeno de orden publicitario sino también cultural del consumo en el que "desde el aspecto del intercambio comunicativo o de datos, que se atribuyen y desde el aspecto del uso que se hace como bien o servicio ${ }^{18}$ ", se establece una nueva forma de comportamiento del consumidor enmarcado por una tendencia cada vez más arraiga en la digitalización de los bienes y servicios a los que desea acceder.

\subsection{De acuerdo a la naturaleza de la campaña publicitaria}

De acuerdo a la naturaleza de la campaña publicitaria, es posible encontrar tres tipos de campaña o intención denominadas dentro del mercado como pull, push y de diálogo continuo o multi pasos. Por efectos de relevancia, sólo se hará alusión a los dos primeros.

16 Perez Tornero, J, M. (2010) “Media Literacy and New Humanism” UNESCO, Institute for Information Techonologies in Education. 2010. 8.

17 Piñuel, Raigada, J L. (1982): "La Publicidad y La Reproducción Social a través de la Producción de Comunicación de Masas" Revista de estudios Políticos (nueva época) N. 29. Pp. 179, 177-196.

18 Íbid. 179. 


\subsubsection{Las campañas "Pull" ${ }^{19 "}$ o campañas de atracción}

Las campañas Pull se caracterizan por una publicidad promovida por cualquier compañía que busque la oferta de bienes y servicios como las compañías de telefonía móvil las cuales, se valen de múltiples códigos para establecer relaciones con sus clientes en términos de uso promocional y consumo promocional además, de la comunicación a través de su branding para informar acerca de un estado de cuenta o servicio, cuya destinación fundamental está y de acuerdo a Mendiz ${ }^{20}$ en referir "marcas, productos (bienes y servicios), a marcas o empresas, u otros asuntos de promoción económica tales como lugares cantantes etc."

En un análisis ${ }^{21}$ que sirvió para llevar al dato empírico el cambio en el mensaje publicitario y la estrategia de que éste se deriva, se partió de un ejercicio de correlación y comparación entre varios usuarios de las distintas compañías telefónicas que prestan sus plataformas para publicitar bienes y servicios en Colombia durante un lapso de tres meses, estableciendo con dicho análisis, la existencia correlacional de una dinámica similar en la manera como estas compañías telefónicas hacen uso de los códigos tanto para informar acerca de los estados de cuenta, así como para ofertar los bienes y servicios de otras entidades.

Dentro del análisis se pudo detectar el uso de 15 códigos distintos, dentro de los cuales, sólo tres de los quince estaban destinados al uso promocional, los doce restantes estaban destinados al consumo promocional. Sin embargo, antes de continuar se hará un alto para explicar en que consiste la distinción entre estos dos tipos de promoción.

Las categorías de uso promocional y consumo promocional han sido a portadas después del análisis como una manera de tipificar las relaciones de mercadeo encaminadas tanto al consumo y de acuerdo a una tipología de mensajes que van desdelos conocidos SMS hasta los Hipertag o Semacodes, así, como al uso de bienes y servicios. En este sentido, se propone en el presente documento estas dos categorías para explicar la relación de las campañas publicitarias en cuanto a la intención de su relación de marketing en tanto relación directa con un segmento abierto o focal, al cual va dirigido el mercado, y con el cual, se pretende de acuerdo a Rodríguez" ${ }^{22}$, que "las personas consciente o inconscientemente, tiendan a exponerse selectivamente a aquellas informaciones de los medios que estén acordes a sus puntos de vista".

19 Pull marketing o de atracción dirige la promoción a los consumidores. Esta diseñada para atraer interés y generar demanda entre los consumidores. La publicidad es dirigida a los consumidores a través de cupones de descuento, recompensas, muestras, demostraciones, mensajes sms, entre otros.

${ }^{20}$ Mendiz, Nogrero A. (2008) "Diferencias Conceptuales entre Publicidad y Propaganda: Una Aproximación Etimológica” Revista Cuestiones Publicitarias, Vol. I, N 12, 2008, pp. 58, 43-61.

${ }^{21}$ El análisis se efectuó entre cinco miembros de una misma familia, que utilizan los servicios telefónicos de las tres compañías más fuertes en el país. El análisis se basó en la clasificación de los códigos utilizados por cada empresa para ofertar sus servicios así, como la publicitación de servicios de otras compañías y agencias dedicadas a la venta de bienes y servicios con fines de entretenimiento.

${ }^{22}$ (2011) "Efectos de las Comunicaciones de Masas de Joseph t. Klapper" Revista Razón y Palabra. Número 75. 5, 1-17. 
De otro lado, al identificar dos tendencias en las campañas publicitarias que puedan contener relaciones de mayor complejidad, se podría establecer una dinámica de relación entre uso/consumo no solo desde una simple relación de recepción de la mediación con fines de mercado, sino también, la puesta en escena de un fenómeno mas complejo en la relación actual entre compañías, medios y mediaciones con fines comerciales en donde la retención selectiva ${ }^{23}$ juega un papel fundamental para lograr la predisposición esperada. De ahí, que su espectro de aplicación pueda servir para una análisis de mayor envergadura dentro del ámbito del M-Advertising.

Volviendo al análisis que sirve de ejemplo para mostrar la relación y alcance de las campañas Pull, se pudo estimar en el análisis y de acuerdo a la nominación propuesta, que el uso promocional de las tres compañías telefónicas, utilizan códigos genéricos tales como: código 869. Código 126, o código 8558. Estos códigos, se basan en la intención de ofrecer a los usuarios de telefonía móvil paquetes de mensajes, minutos adicionales, servicios de tipo doméstico entre muchos otros, de una amplia gama que se oferta como bienes y algunos de servicios y soporte de asistencia. En cuanto al consumo promocional, se pudo detectar la oferta de servicios que van desde backtones, hasta promoción de artistas, eventos y farándula. Mensajes que a diferencia del uso promocional no busca la posición de una marca, sino el consumo de segmentos y servicios de moda.

\subsubsection{Las campañas denominadas "Push" ${ }^{24}$ " o campañas de información}

Son campañas que buscan impactar segmentos, se compone de una base da datos que operan a través del denominado Permission Marketing ${ }^{25}$ que a diferencia de las campañas pull, no basa su estrategia sobre un mercado target, sino en el denominado marketing one to one. Sin embargo, la diferencia radical está en que mientras el pull marketing no discrimina totalmente la oferta del servicio al grupo destino, en el push marketing, debe haber un contacto previo con el cliente que permita a través de un convenio previo el envío de portafolios, promociones y oferta de bienes y servicios específicos. Es el caso, de las tiendas virtuales que mediante motores de búsqueda crean perfiles de potenciales clientes y mediante registro previo, envían según los perfiles de búsqueda promociones y bienes o servicios semejantes a los que el potencial cliente estuvo buscando.

\footnotetext{
23 Íbid. P.6

${ }^{24}$ Push marketing o de empuje es cuando los esfuerzos de mercadeo o promoción van dirigidos a los canales de distribución como lo serían los detallistas. Esta estrategia pretende que al canal de distribución le interese el producto y lo quiera comprar y almacenar para tenerlo disponible para el consumidor. Se basa en la venta y exhibición comercial y la utilizan los fabricantes que desean posicionar su marca.

${ }_{25}$ Se basa en el principio de que los consumidores primero deben dar su permiso (opt-in) en vez de rechazar (opt-out) después de que la publicidad haya sido enviada. Todo ello logra un uso más eficiente de recursos, ya que los promocionales no son enviados a personas que no están interesadas en el producto. Es una técnica basada de alguna manera en la orientación del marketing personal, es decir en el concepto de marketing uno-a-uno, a diferencia de las conceptos masivos o a escala como la segmentación del mercado o mercado target.
} 
No obstante, y de acuerdo a García Canclini citado por León Duarte 26 "la ubicación del consumo como parte del ciclo de producción y circulación de los bienes, (...) permite hacer visibles aspectos más complejos (...) lo que desencadena en la recepción, apropiación y las audiencias o usos". Así pues, lo que las campañas tipo Pull y Push establecen, es cierto modo de consumo de tipo cultural, en el que ya no se busca un valor de cambio, sino una apropiación de valor simbólico ${ }^{27}$. De ahí y como lo plantea Menucci ${ }^{28}$ que el vínculo creado desde el valor simbólico asignado al producto o servicio esperado del mismo, sea más que un vínculo, "sea una relación basada en confianza", donde a partir de la comunicación se logra cierto grado de manipulación simbólica y gestión estratégica de las conductas.

De ahí que las campañas tipo Pull y Push, esten diseñadas básicamente para usuarios intensivos de dispositivos móviles o heavy user; usuarios, para quienes el móvil constituye su principal fuente de información y posibilidad de vincular una conducta de uso/consumo. Estas campañas, como su nombre lo indica, están diseñadas para una oferta de servicios paso a paso que permiten la publicitación de bienes y servicios de manera detallada y secuencial, por ejemplo como las ventas por portales como ebuy, o best buy, mercado libre o amazon, portales de ventas de bienes y servicios que permiten un vínculo directo a través del uso de dispositivos móviles para seguir tanto la venta así, como para concretar las operaciones de lo que se oferta.

\subsection{De acuerdo a los soportes del medio comunicativo utilizado para la campaña}

Dentro del rango de mediaciones utilizadas para hacer efectiva una campaña publicitaria, se pueden contar alrededor de diez soportes de mediación los cuales han sido clasificados de acuerdo a su intención en un artículo de la profesora Sandra Martínez Acosta ${ }^{29}$ en 2006. Estos soportes varían desde mensajes de texto sencillos, hasta mensajes con soporte audiovisual dependiendo del medio tecnológico disponible.

Entre los soportes utilizados en la mediación de la publicitación de bienes y servicios se encuentran:

1. mensajes SMS

2. mensajes MMS

3. mensajes WAP

4. mensajes de VOZ/PODCAST

5. mensajes VODCAST/CONTENIDOS DE VIDEO

${ }^{26}$ Leon, Duarte, G. (2002): “Teorías e Investigación de la Comunicación en América Latina Situación Actual” Revista Ámbitos, Número 7-8, 2 , 37, 19-47.

27 Íbid. 37.

${ }^{28}$ Manucci, M. (2005): “Innovación y Estrategia en Comunicaciones de Marketing” III congreso internacional de comunicación organizacional. Pontificia Universidad Javeriana, Cali-Colombia. 3, 1-21.

${ }^{29}$ Acosta, Martinez, S. (2006) "La publicidad móvil. El marketing en los nuevos medios". Publicidad y Marketing, IX congreso IBERCOM. Sevilla-Cádiz, 4, 1-9. 

6. juegos
7. servicios geo-localización
8. publicidad above the line
9. códigos bidimensionales, HIPERTAG o SEMACODES
10. reconocimiento de imágenes

Para el caso de los mensajes SMS, éstos son de una cobertura más amplia dado su formato simple, y compatibilidad universal con cualquier dispositivo móvil de alta o baja gama, en cuanto a las características, el formato SMS soporta una limite de 160 caracteres, lo que ha hecho que siga siendo uno de las más económicos y usados para mediar bienes y servicios.

El soporte MMS a pesar de ser más atractivo en su presentación en comparación con los SMS, resulta poco utilizado para publicitar bienes y servicios, por un lado, porque aún no se ha optimizado uso habitual de la publicidad audiovisual que vaya de lo medios masivos al bolsillo del usuario. Si bien, en la actualidad hay una buena oferta de dispositivos de telefonía móvil con capacidad para recepcionar información el formato MMS, al parecer el uso y consumo es restringido a unos pocos grupos con intereses específicos.

Los soportes WAP, son ampliamente utilizados entre usuarios con dispositivos móviles que posean una conexión a Internet vía wifi o dispongan de un plan de datos. Sin embargo, presenta la misma restricción del soporte MMS, ya que no siempre los usuarios siguen los enlaces vinculados a los mensajes a pesar, de tener mejor aceptación que los MMS. En cuanto a los soportes podcast y vodcast, a pesar de estar en pleno desarrollo se estima la necesidad de un cambio de mentalidad en la relación usuario/ medio, que permita una mayor optimización para el alcance e intención de la mediación.

Entre las múltiples ventajas que este nuevo formato o medio de comunicación comercial posee está, la gran ventaja de estar codificado de acuerdo a necesidades específicas de las compañías que los estructuran, personalizando el mensaje de acuerdo a la demanda del mercado caracterizada por consumidores en movimiento o M-Averts denominación que hace parte del llamado "M-Commerce" es decir, de aquel grupo de publicidad caracterizada por el envío único de mensajes de manera personalizada, y directa a través de dispositivos móviles.

Sin embargo, en comparación con los modelos de comunicación publicitaria clásica, el marketing interactivo representa una forma de transición del objeto de la mediación. Una transición, que ha surgido gracias a un fenómeno de orden social en contacto con patrones de tecnología personalizada.

\section{Conclusión}

Así pues, mientras que en los medios de masa se pretendía tres ejes fundamentales que iban del formar, informar hasta el divertir la mayor cantidad de audiencias posibles a partir de emisiones generales y destinadas a grandes porciones de la población. Con el nacimiento de la web 2.0 y con el desarrollo de plataformas tecnológicas diseñadas para optimizar sus aplicaciones, se sucedió una transformación en el eje del uso/valor, que en el margen de la teoría neoclásica de la economía bajo la óptica del utilitarismo 
marginalista, estableció una relación entre uso y consumo, que lentamente fue mutando hacia otras formas más complejas y personalizadas del mercado al punto, de llegar a formularse una concepción sobre las nuevas formas de consumo a partir de un individualismo en el que es posible encontrar variables que contribuyen no solo a la comprensión de los cambios en el uso consumo bajo una forma de necesidad básica, pasando por formas de bienestar hasta llegar a usos expresivos y simbólicos de los medios portátiles destinados a la persuasión del comportamiento a través de la cada vez mayor sofisticación M-Adverts ello, desde la identificación de los grupos de referencia $\mathrm{y}$ grupos de pertenencia.

Dentro de este denominado consumo individualista, se pueden identificar cuatro factores de incidencia que llevan a la formación y constitución de una conducta de consumo. Estos factores bien pueden ser clasificados en cuatro determinantes:

1. Conducta de Consumo.

2. Factores Psicológicos o Formas de Conducta de Consumo.

3. Conductas Económicas Afectivas y Expresivas.

4. Contacto del Consumidor con los tipos de Marketing Organizacional.

5. Transición del uso/valor del consumo hacia el uso expresivo y simbólico del consumo.

Del primer factor, se desprende una serie de variables que han llevado a lo largo de la historia a configuraciones diversas del uso/valor y del uso/de cambio del consumo. Dentro de estas variables en la formación de una conducta de consumo se dan factores culturales o simbólicos, los cuales, van ligados a los imaginarios sobre bienestar, necesidad, aspiraciones y actitudes en las dinámicas de grupo, de otro lado, surgen patrones de comportamiento o factores psicológicos determinados por los mismos imaginarios sobre necesidad y bienestar así, como por las motivaciones que lleven a su realización. Por último, se encuentran las fuerzas, el mercado o factores de transición del uso/valor, uso/de cambio y uso/simbólico de lo que se consume identificable, a partir de las fluctuaciones del mercado, la cantidad de ingresos, los precios y los haberes. En consecuencia, el cambio del marketing en sentido lapso al mobile marketing, se podría pensar como el surgimiento de una nueva forma de cultura publicitaria en la que el espectador se reconoce como consumidor en interacción continua con múltiples mensajes publicitarios que se han ido constituyendo en formas o productos intangibles y culturales de consumo.

\section{Bibliografía}

Barbero, M Y Tellez, M. P. (2006): "Los Estudios de Recepción y Consumo en Colombia”. Revista Diálogos de Comunicación. 57-69 http://www.researchgate. net/publication/28225048_Los_estudios_de_recepcin_y_consumo_en_Colombia Web visitada el 28/05/2014.

Carrillo, M. (2005): "La Interactividad: un Reto para la Publicidad en el Entorno Digital". Revista Zer. 9-24. http://www.ehu.es/zer/hemeroteca/pdfs/zer18-01carrillo.pdf Web visitada el 28/05/2014. 
Ceruelo, C Y Gutierrez, A. (2003): "Eficacia de la publicidad emocional. Un estudio comparativo entre la ejecución de tipo emocional e informativa". Repositorio documental Gredos. http://hdl.handle.net/10366/75262. Web visitada 26/05/2014.

Costa, Martinez, S. (2006): "La publicidad móvil. El marketing en los nuevos medios". Publicidad y Marketing, IX congreso IBERCOM. Sevilla-Cádiz. 1-9. http:// portaldocomerciante.xunta.es/Archivos/ArchivosImpBiblioteca/publicidad_movil. pdf Web visitada el 27/05/2014.

Del Pino Romero C. (2010): "La Internet y los Nuevos Consumidores: el Nuevo Modelo Publicitario". Revista telos Dossier, 55-82. http://sociedadinformacion. fundacion.telefonica.com/url-direct/pdf-generator?tipoContenido=articuloTelos\&i $\mathrm{dContenido}=2010020215530001$ \&idioma $=$ es eb visitada el 28/05/2014.

GALÁn, E Y Pino, C. (2010): "Internet y los nuevos consumidores. El nuevo modelo publicitario". Revista de pensamiento sobre comunicación, tecnología y sociedad Telos. 80-96, http://telos.fundaciontelefonica.com/url-direct/pdf-generator?tipoCon tenido $=$ articuloTelos\&idContenido $=2010020215530001 \&$ idioma $=$ es, Web visitada el 27/05/2014.

Gomez Tinoco, A. (2010): "El Mobile Marketing como Estrategia de Comunicación". Rrevista Icono 14, Vol. $8 \mathrm{~N}^{\circ} 1$, http://www.icono14.net/Num.-15.-Interactividad/ mobile-marketing Web visitada el 28/05/2014.

Gomez Rosado, D, (2004): "Publicidad Interactiva". Revista Mosaic, tecnologías y comunicación multimedia, 3-31. http://hdl.handle.net/10609/9165.Web visitada el 28/05/2014.

Gonzales OÑate, C. (2008): "Estrategia de Comunicación Publicitaria para el Lanzamiento de una Marca" http://www.bocc.ubi.pt/pag/gonzalez-onate-cristinaestrategias-de-comunicacion-publicitaria.pdf Web visitada el 26/05/2014

RodriguÉz-Polo, R. (2011): "Efectos de las Comunicaciones de Masas de Joseph t. Klapper". Revista Razón y Palabra. Número 75. www.razonypalabra.org.mx. Web visitada el 28/05/2014.

Leon, Duarte, G. (2002): "Teorías e Investigación de la Comunicación en América Latina Situación Actual”. Revista Ámbitos, $N^{\circ} 7-8,2^{\circ}$, pp. 19-47. http://grupo. us.es/grehcco/ambitos07-08/duarte.pdf. Web visitada el 28/05/2014.

Manucci, M. (2005): "Innovación y Estrategia en Comunicaciones de Marketing". III congreso internacional de comunicación organizacional. Pontificia Universidad Javeriana, Cali-Colombia. http://d3ds4oy $7 \mathrm{~g} 1$ wrqq.cloudfront.net/ comunicacion-organizacional/myfiles/Innovacion $\% 20 \mathrm{y} \% 20$ estrategia $\% 20 \mathrm{en} \% 20$ comunicaciones\%20de\%20marketing\%20Marcelo\%20Manucci.pdf Web visitada el $28 / 05 / 2014$.

Mendiz, Nogrero A. (2008): "Diferencias Conceptuales entre Publicidad y Propaganda: Una Aproximación Etimológica". Revista Cuestiones Publicitarias, Vol. I, No 12, 2008, pp. 43-61. http://www.maecei.es/pdf/n12/articulos/Diferencias_ conceptuales entre publicidad y propaganda.pdf

mana Code For Responsible Mobile Marketing. (2003): "A code of conduct and guidelines to best practice", www.mmaglobal.co.uk Web visitada el 27/05/2014. 
Perez Tornero, J, M. (2010): "Media Literacy and New Humanism" UNESCO, Institute for Informatio9n Techonologies in Education. http://unesdoc.unesco.org/ images/0019/001921/192134e.pdf Web visitada el 28/05/2014.

Piñuel, Raigada, J L. (1982): “La Publicidad y La Reproducción Social a través de la Producción de Comunicación de Masas”. Revista de estudios Políticos (nueva época) $\mathrm{N}^{\circ}$. 29. Pp. 177-196. http://www.razonypalabra.org.mx/anteriores/n12/ persp12.html Web visitada el 28/05/2014.

ROMERA SAN N. C. (2004): "Publicidad Corporativa y Cultura Cotidiana". http://www. comminit.com/la/node/150542 Web visitada el 28/05/2014. 